\title{
Force Statistics and Correlations in Dense Granular Packings
}

\author{
Micha-Klaus Müller ${ }^{\mathrm{a}}$, Stefan Luding ${ }^{\mathrm{a}}$, Thorsten Pöschel ${ }^{\mathrm{b}, \mathrm{c}}$ \\ ${ }^{a}$ Multi Scale Mechanics, CTW, UTwente, P.O.Box 217, 7500 AE Enschede, Netherlands \\ ${ }^{b}$ Universität Erlangen-Nürnberg, Institute for Multiscale Simulation, Erlangen, Germany \\ ${ }^{c}$ Cluster of Excellence 'Engineering of Advanced Materials', Friedrich-Alexander-University, \\ Erlangen, Germany
}

\begin{abstract}
In dense, static, polydisperse granular media under isotropic pressure, the probability density and the correlations of particle-wall contact forces are studied. Furthermore, the probability density functions of the populations of pressures measured with different sized circular pressure cells is examined. The questions answered are: (i) What is the number of contacts that has to be considered so that the measured pressure lies within a certain error margin from its expectation value? (ii) What is the statistics of the pressure probability density as function of the size of the pressure cell? Astonishing non-random correlations between contact forces are evidenced that lead to a rapid decay of the width of the distribution and range at least 10 to 15 particle diameters. Finally, an experiment is proposed to tackle and better understand this issue.

Keywords: Force and stress correlations, DEM simulations, dense static granular packings, pressure cells
\end{abstract}

PACS: 45.70, 47.50+d, 51.10.+y

\section{Introduction}

One of the open issues in the field of disordered, random systems like dense, static granular packings, is the probability density of the contact forces and

Email address: s.luding@utwente.nl (Stefan Luding) 
their possible long range correlations. There is common agreement that the probability for large forces decays exponentially $[1,2,3,4,5,6,7,8,9,10,11$, $12,13,14,15]$ but the small forces are much harder to measure $[2,16,17]$, so that there is still ongoing discussion about the shape of the probability density for small forces, possible correlations between the forces, and a predictive model for the force propagation inside dense packings of frictional particles [18].

Furthermore, it is observed that the deformation of particle systems is not affine in general, but displays finite distance correlations which are assumed to increase when approaching the jamming transition $[6,19,20,21,22,23,24$, 25, 26, 27]. However, the issue whether these correlations (and possibly anticorrelations due to vortex formation) depend on the system size [28] or not $[29,30]$ is not completely resolved yet.

Since the forces that granular particles exert onto their confinement (walls) strongly fluctuate from one particle to the next, so does also the local pressure. When the pressure on the wall is measured with a circular pressure cell, performing many independent measurements, one obtains a probability density of the measured pressure, with its first moment approaching the mean pressure, and the standard deviation decreasing with increasing cell size. While the case of uncorrelated forces is rather behaving as expected, the case of subtly correlated forces in granular packings leads to interesting results and probably can be understood with advanced statistical methods [34], which can e.g. account for contact number fluctuations. Data on stress fluctuations in sheared systems of frictional particles were reported in Refs. [31, 32]. Miller et al. [31] observed that the width of the distribution was rather independent of the particle size and thus weakly dependent on the number of particles contributing to the stress measured on their pressure cell. They attributed this to the fact that force chains rather than single particles are carriying most of the load so that they become responsible for the statistics rather than the number of particle contacts. In our study we will therefore simplify by switching off friction and shear and, instead of varying particle size, we measure the pressure at the walls with pressure-cells of different sizes. Baran and Kondic [32], on the other hand, observed that the 
width of the distribution decays slower than for un-correlated forces for larger averaging times and attribute this to the absence of zero-force contacts. Their system, however, was sheared in the collisional and thus dynamic regime, unlike the quasi-static isotropically stressed situation considered in this study.

A local measurement of the wall pressure can be far away from the total pressure (or the mean, representative pressure), unless "enough" particles are contained in the pressure cell. Besides the question, how much "enough" is, also the question of the behavior of the width of the pressure probability density is examined in this study. The reason to examine the pressure distribution instead of the force distribution is that the former is much easier to access experimentally, as will be outlined at the end of this paper.

In general, more knowledge on the force- and pressure-density functions is needed for the understanding of pressure measurements aiming, for example, at a safe design of containers of granular materials such as silos.

The simplest model for the force probability density function is the so-called $q$-model, introduced 1995 by Liu, et al. [16, 2], that describes the occurrence of force chains based on uncorrelated probabilistic assumptions. Even though there are many more advanced models available that take into account the disorder and the geometric packings of granular media, see e.g. Refs. [14, 15], we use the $q$-model as starting point for convenience. In a dense packing of discrete particles, the contact forces that one particle in a certain layer feels from above plus its weight, determines the sum of the contact forces on the particles below. The magnitude of the two forces at the two contacts below, are the fractions $q$ and $1-q$ of the sum. In general, the weights can also depend on neighboring sites and contacts can open and close. The mean field approximation, however, neglects these dependencies and thus simplifies the model vastly. Eq. (1) gives us the normalized, scaled density function of the inter-particle forces $f_{*}=f /\langle f\rangle$, predicted by the $q$-model:

$$
p_{q}\left(f_{*}\right)=\frac{C^{C}}{(C-1) !} f_{*}^{C-1} \exp \left(-C f_{*}\right),
$$

where $C$ is the number of the neighboring particles (below or above). The 
weakness of the $q$-model is the improper prediction of the probability to find small forces, see Fig. 1.

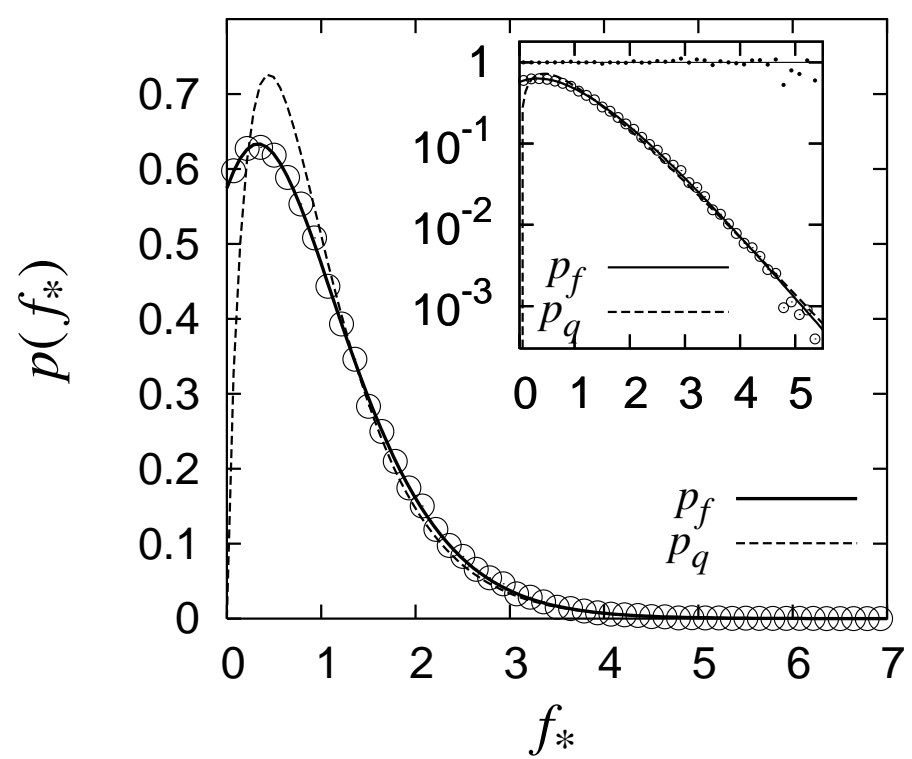

Figure 1: Normalized probability density $p\left(f_{*}\right)$ plotted against the normalized force $f_{*}=$ $f /\langle f\rangle$. The circles are simulation data, and the dashed and solid lines are Eqs. (1) and (2), with $C=1.8$, respectively. The inset shows the same data in log-scale, together with the "quality factor", i.e. the simulation data divided by the fit-function as dots around unity; the fit has deviations less than two per-cent for $f_{*}<3$ and less than ten per-cent for $f_{*}<5$. The $q$-model, Eq. (1), is invalid for $f_{*}<0.2$ and has varying deviations of about ten per-cent for $0.2<f_{*}<5$.

A function, that provides an excellent fit to the simulation data is

$$
p_{f}\left(f_{*}\right)=\left(1-a \exp \left(-\frac{\left(f_{*}+b\right)^{2}}{8}\right)\right) c \exp \left(-d f_{*}\right),
$$

with the fit-parameters $a=0.983 \pm 0.003, b=0.56 \pm 0.05, c=1.80 \pm 0.02$, and $d=10.4 \pm 0.7$. Note that a similar function was found experimentally, see Refs. [35, 36]. The number of parameters can be reduced by using the normalization relations $\int p_{f} d f=1$ and $\int f p_{f} d f=1^{1}$. The fit-parameters for

\footnotetext{
${ }^{1}$ the factor 8 in the denominator leads to some better fit-quality by stretching the Gaussian correction function
} 


\begin{tabular}{l|l|l|l|l}
\hline$\nu$ & $a$ & $b$ & $c$ & $d$ \\
\hline 0.80 & 0.995298 & 0.477783 & 14.089 & 1.91749 \\
\hline 0.70 & 0.971913 & 0.68069 & 7.72909 & 1.68119 \\
\hline 0.68 & 1.00345 & 1.05915 & 5.127 & 1.54689 \\
\hline 0.66 & 0.970768 & 1.11105 & 4.25643 & 1.47278 \\
\hline 0.64 & 0.902126 & 0.507015 & 6.3781 & 1.57989 \\
\hline$e[\%]$ & $1-4$ & $15-23$ & $11-20$ & $2-4$ \\
\hline
\end{tabular}

Table 1: Fit results for different volume fractions $\nu$, where the row $e$ contains the typical relative error of the respective fit-parameter.

different densities are given in table 1.

\section{Theory}



WALL

Figure 2: The forces of particles that touch the walls fluctuate around a mean value.

Let us consider a cubical container filled with granular material under isotropic, hydrostatic pressure. Each particle $i$ of the $N_{w}$ particles that touch a wall exerts a force $f_{i}$ (see Fig. 2) on the wall and contributes to a (finite: $1 \leq i \leq N_{w}$ ) population of forces $p(f)$ with mean $\mu_{w}=\langle f\rangle$ and standard deviation $\sigma_{w}$, where the subscript $w$ refers to the population of all wall-particle forces. If we select one sample $j$ of size $n$ out of this population by applying a circular sensitive area $D(R)=\pi R^{2}$ that includes the $n$ forces that are acting on this area, we obtain the pressure

$$
P_{j}(R)=P_{j}\left(n_{j}(R)\right)=\frac{(1 / n) \sum_{j=1}^{n} f_{j}}{D(R) / n}=(1 / n) \sum_{j=1}^{n} f_{j} / D_{1},
$$


with the area per particle, $D_{1}=D(R) / n$, and $n=n_{j}(R)$. Note that such areas have to be selected such that their center - on the selected wall - is at least a distance $R$ away from any other wall. Taking many samples, $m \gg 1$, will result in a population of pressure values with probability density $p_{R}(P)$ around the mean $\mu_{P}=\left\langle P_{j}(R)\right\rangle=(1 / m) \sum_{j=1}^{m} P_{j}(R)$, with standard deviation

$$
\sigma_{P}=\sqrt{\left\langle P_{j}^{2}(R)\right\rangle-\mu_{P}^{2}}
$$

where the subscript $P$ refers to the population of pressures.

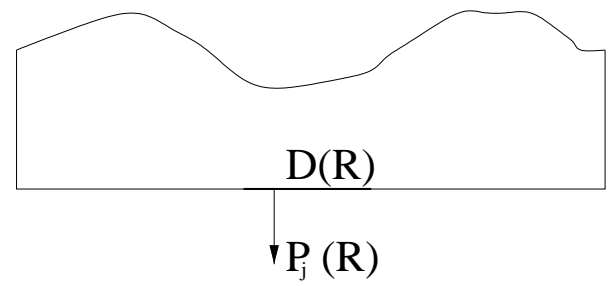

Figure 3: The average force of sample $j$, divided by the sensitive area $D(R) / n$ per particle, of this detector, leads to a pressure $P_{j}(R)$ corresponding to one measurement $j$.

\subsection{Central Limit Theorem}

According to the central limit theorem (CLT), and for the corresponding assumptions, the probability density function $p_{R}(P)$ of our population of samples $P_{j}(R)$ provides the same expectation value as for the original population $\mu_{P} \approx \sum_{i=1}^{N_{w}} f_{i} / A$, where $A$ denotes the total surface of the confinement and the sum goes over all particles in the system touching the walls ${ }^{2}$. The CLT also tells us that the probability density function becomes more and more Gaussian:

$$
g_{R}(P)=\frac{1}{\sqrt{2 \pi} \sigma_{P}} \exp \left[-\frac{1}{2}\left(\frac{P-\mu_{P}}{\sigma_{P}}\right)^{2}\right],
$$

the larger we chose $n$, i.e., by increasing the detector size $R$. The standard deviation then equals $\sigma_{P}=\sigma_{P}(R)=\left(n(R) \sigma_{w}\right) /(D(R) \sqrt{n(R)})=\sigma_{w} /\left(D_{1} \sqrt{n(R)}\right)=$ :

\footnotetext{
${ }^{2}$ This is valid under the assumption that all wall-particles are similar, i.e. there is no inhomogeneity in the forces on the wall particles, e.g. as function of distance from another wall in the edges of the cuboid.
} 
$P_{f} / \sqrt{n}$, with the pressure $P_{f}$ that corresponds to the standard deviation of the force density function scaled by the area of the pressure cell per particle.

\subsection{Confidence Intervals}

Integration from $-\infty$ to $+\infty$ of the normalized distributions $p_{R}(P)$ and $g_{R}(P)$ gives unity. Now, to gain more advanced statistical predictions about the pressure distribution, let us consider a lower and an upper integration limit $z_{\alpha / 2}<\mu_{P}$ and $z_{1-\alpha / 2}>\mu_{P}$, respectively, such that the integral over Eq. (4) equals

$$
\begin{aligned}
1-\alpha=\int_{z_{\alpha / 2}}^{z_{1-\alpha / 2}} g_{R}(P) d P & =1-\int_{-\infty}^{z_{\alpha / 2}} g_{R}(P) d P-\int_{z_{1-\alpha / 2}}^{\infty} g_{R}(P) d P \\
& =\frac{1}{2}\left[\operatorname{erf}\left(\frac{z_{1-\alpha / 2}-\mu_{P}}{\sqrt{2} \sigma_{P}}\right)+\operatorname{erf}\left(\frac{\mu_{P}-z_{\alpha / 2}}{\sqrt{2} \sigma_{P}}\right)\right] .
\end{aligned}
$$

If the integration limits are chosen such that a fraction $\alpha / 2$ lies outside of the integration range, both to the left and the right, the integration limits correspond to the confidence interval $2 \delta_{\alpha}=z_{1-\alpha / 2}-z_{\alpha / 2}$; a fraction $1-\alpha$ of the $n$ measurements $P_{j}$ lies within the confidence interval.

Keeping $n=$ const. and considering $\alpha=0$ (probability for finding the measured value in-between our limits then equals 1 ), we expect that the interval of confidence tends to infinity. On the other hand, for $\alpha=1$ (probability vanishes), one expects $\delta_{\alpha} \rightarrow 0$.

Due to the symmetry of the Gaussian distribution, one can compute

$$
\delta_{\alpha}:=z_{1-\alpha / 2}-\mu_{P}=\mu_{P}-z_{\alpha / 2}
$$

explicitly, using the relation: $\operatorname{ierf}(1-\alpha)=\delta_{\alpha} /\left(\sqrt{2} \sigma_{P}\right)=\delta_{\alpha} \sqrt{n} /\left(\sqrt{2} P_{f}\right)$ such that:

$$
n=2\left(P_{f} / \delta_{\alpha}\right)^{2} \operatorname{ierf}^{2}(1-\alpha)
$$

where the pressure $P_{f}=n(R) \sigma_{w} / D(R)=\sigma_{w} / D_{1}$ is, of course, a constant for given geometry and $p(f)$. The general equation (7) gives us information about how many particles $n$ need to be considered in a measuring process, so that a 
measurement $P_{j}(R)$ lies in-between the error margin $\pm \delta_{\alpha}$ with probability $1-\alpha$. Note, that $\delta_{\alpha}$ and $n$ depend on the size $R$ of the sensitive area $\delta_{\alpha} \propto 1 / \sqrt{n(R)} \propto$ $1 / R$.

\subsection{Explicit predictions}

\subsection{1. q-model}

Now we use the distribution predicted by the $q$-model for the analysis. The first two moments obtained from Eq. (1) are $\bar{f}=\langle f\rangle$ and $\overline{f^{2}}=\frac{C+1}{C}\langle f\rangle^{2}$, which leads to the standard deviation $p_{q}(f)$, namely $\sigma_{f}^{q}=\left(\overline{f^{2}}-\bar{f}^{2}\right)^{1 / 2}=\sqrt{1 / C}\langle f\rangle$. Considering Eq. (1) as a finite population and applying the CLT by taking many samples, one can then replace $P_{f}^{2}$ in Eq. (7) to get

$$
n^{(q)}=\frac{2}{C}\left(\frac{\langle f\rangle}{D(R) / n}\right)^{2} \operatorname{ierf}^{2}(1-\alpha) \frac{1}{\delta_{\alpha}^{2}}=(2 / C) \operatorname{ierf}^{2}(1-\alpha)\left(P_{f} / \delta_{\alpha}\right)^{2} .
$$

\subsubsection{Best Fit}

Now we use the distribution as obtained by our fit to the data for the analysis. The first two moments obtained from Eq. (2) are $\bar{f} \approx\langle f\rangle, \overline{f^{2}}=1.61\langle f\rangle^{2}$ and the corresponding standard deviation can be computed to $\sigma_{f}^{f i t}=0.78\langle f\rangle$. In comparison with these results the $q$-model provides $\sigma_{f}^{q}=0.76\langle f\rangle$ for a dense granular packing with $C=1.8$ corresponding to a special geometry.

\section{Simulation}

\subsection{The System}

The systems studied in the following contain $N$ perfectly spherical particles with radii $r_{i}$ drawn from a homogeneous distribution $r_{i} \in\left[r_{\min }, r_{\max }\right]$, where we used $r_{\max } / r_{\min }=2$ and 3 , and the mass $(4 / 3) \pi r_{i}^{3} \rho$, where $\rho$ represents the uniform material density of the particles. Since the mass is not relevant in the static limit, we refer to density only with the dimensionless volume fraction $\nu_{f}=\frac{4}{3 V} \pi \sum_{i}^{N} r_{i}^{3}$, with volume $V=\prod_{\beta=1}^{3} L_{\beta}$, where $L_{\beta}$ denotes the length of the simulation volume in direction $\beta \in(x=1, y=2, z=3)$. All of the simulations were done in a cuboid volume which is limited by walls that repell 


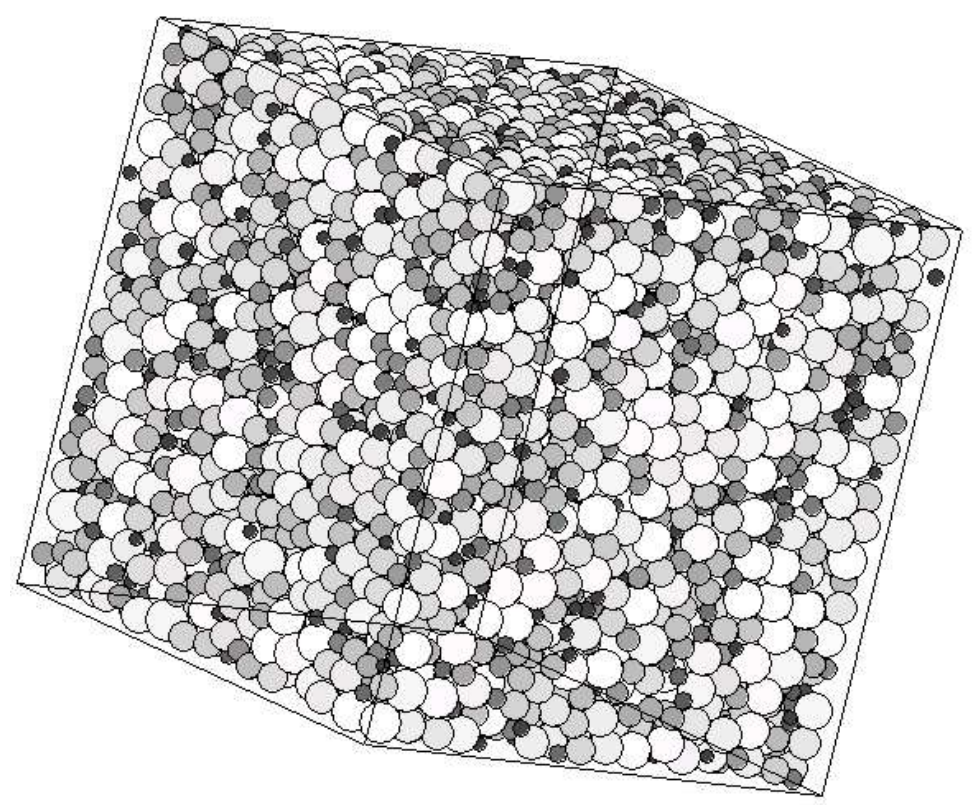

Figure 4: Snapshot of a dense, polydisperse assembly of $N=8000$ particles confined in a cuboid. The volume fraction here is $\nu \approx 0.7$ and the particle sizes are grey scaled (bright particles are big, dark particles are small).

touching particles. Fig. 4 gives an example of a typical static and dense granular sample, while Fig. 5 shows a representative subset of particles that touch a wall (Left) and the corresponding magnitudes of forces (Right), quantified by the radius of the circles.

\subsection{Molecular Dynamics}

The simulations were performed by means of a molecular dynamics (MD) code in three dimensions, without tangential forces like friction. MD simulations are characterized by discretizing time into timesteps $\Delta t$ and solving the Newton's equations of motion for each particle. In each integration step, the new position of the particles is computed from its previous and present positions 

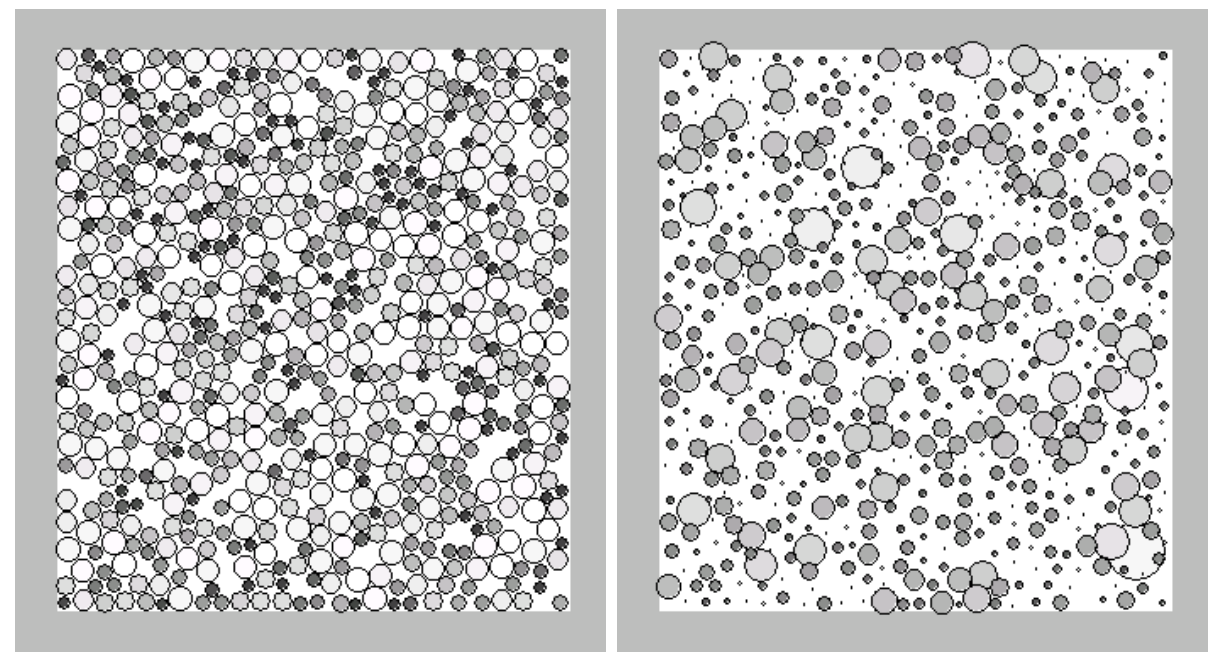

Figure 5: View of one of the walls for a packing with $N=20000$ particles with $\nu \approx 0.67$. (Left) Each circle is a particle in contact with the wall; the color coding is the same as in Fig. 4. (Right) Same data as (left), but here each circle radius is scaled with the force exerted by the particle on the wall. (Big, bright circles correspond to large forces, whereas small, dark circles correspond to small forces on the wall).

and accelerations due to forces currently acting on it [37]. MD is also referred to as discrete element method (DEM) or as soft-sphere model, i.e. the repulsive forces $f^{n}$ normal to the plane through the point of contact depend on the overlaps of the spheres, that replace the contact deformation. The linear springdashpot (LSD), the non-linear Hertzian model and a hysteretic force model can be used [38], among many others. For particle-wall contacts, the same spring constant were used as for contacts between particles. Here, only the LSD results are discussed because no qualitative differences could be evidenced for the different contact laws.

\subsection{Initial Configurations and Relaxation}

As initial configurations, $N=8000$ or $N=20000$ poly-disperse particles with random initial velocities were placed on a regular cubic lattice with low total density. Due to the free space between the particles, the initial order is forgotten and the particles dissipate energy during collisions. Eventually, the 
dense, relaxed and disordered granular packing is obtained by either applying hydrostatic pressure on the walls or by growing the particles. Due to the dissipative nature of the contact law, energy is dissipated and the system reaches a static configuration, where we use as criterion the ratio of kinetic and potential energy, $E_{\text {kin }} / E_{\text {pot }}=\epsilon$, with $\epsilon \leq 10^{-7}$. If $\epsilon$ is small, the particles are typically almost at rest and the major contribution to the total energy stems from the contact potential energy between particles and between particles and the walls. Since the results were identical for the two preparation procedures, we only mention that there are more alternative ways of achieving a static packing, see [39], which is far from the scope of this study. Furthermore, we do not consider friction, since friction makes the packing structure dependent on the history and on details of the preparation procedure.

\section{Results}

In this section, we present the results concerning force probability density functions. When scaled by their respective mean, the particle-particle and particle-wall force probability density functions were identical - within the considerably larger fluctuations of the latter. The larger fluctuations are due to the smaller number of wall contacts as compared to the bulk contacts. Since we are interested in the experimentally accessible pressure measured at the wall, we will not present bulk data in the following, except for some qualitative comparison between wall and bulk results.

\subsection{Width of probability distributions}

When a circular measuring area with radius $R$ is put around each wallparticle (for all wall particles with distance larger than $R_{\max }$ from any other wall), one obtains a set of $P_{j}(R)$ values and, from these, can compute the mean values and standard deviations. A typical set of wall-particles is shown in Fig. 5. Note that $R_{\max }$ is introduced, so that the same set of wall-particles is used for the computation independent of $R$ and, if $R_{\max } / r_{\max }$ becomes larger than 
12-13, the statistics becomes bad, since too few particles close to the center of the wall are taken into account as the centers of pressure cells.

For each sample with given $R$, from the corresponding pressures $P_{j}(R)$, the histograms are obtained, as shown in Fig. 6 for three different $R$-values. The larger the cell size, the more the pressure density function appears Gaussian and, as Fig. 7 shows, the smaller the standard deviation $\sigma_{P}$ becomes. Note, however, the interesting fact, that the decay of $\sigma_{P}$ is steeper than the simple relation $\sigma_{P} \propto 1 / R$, expected from the central limit theorem.


Figure 6: Probability density for the pressures from many samples with sizes $R_{*}=R / r_{\max }=$ 1.0, 2.5, and 5.0. Points are simulation data and lines correspond to Eq. (4) The top panel shows the same data as the bottom panel, where the latter has a logarithmic vertical axis. 


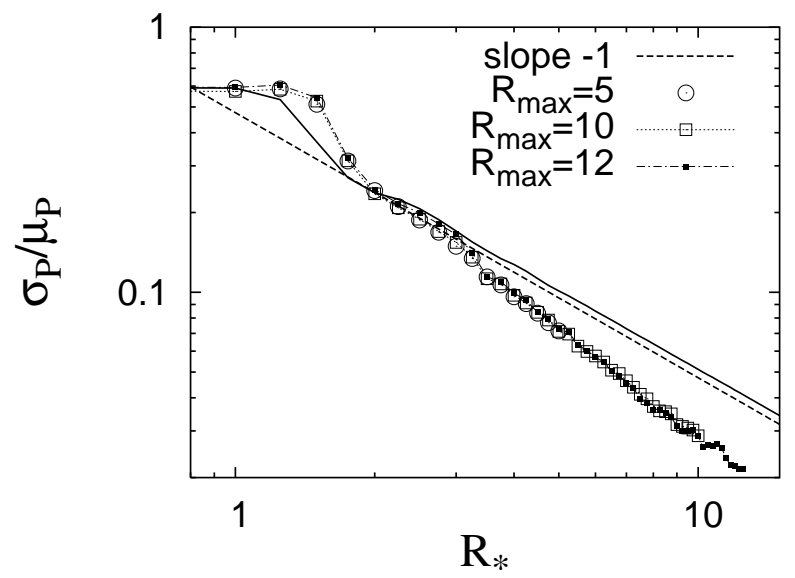

Figure 7: Scaled standard deviation $\sigma_{P} / \mu_{P}$ plotted against the size of the cells $R_{*}$ for different $R_{\max }$. The symbols are simulation data, while the solid and the dashed lines correspond to $\sigma_{P} / \mu_{P}=\sqrt{n} \sigma_{w} /\left(\pi R^{2} \mu_{P}\right)$ and $\sigma_{P} / \mu_{P} \propto 1 / R_{*}$, respectively.

Fig. 7 also contains steps for small cell sizes due to integer jumps in the particle number $n(R)$. For larger $R$, the change of $\sigma_{P}$ becomes smooth and independent of $R_{*}$. For every kind of distribution of the population we should get - according to CLT - a Gaussian distribution of the $P_{j}(R)$ around the population mean. Eq. (7) is a consequence of these simplifications. But our simulations show $\langle n\rangle \propto \delta_{\alpha}^{-1.5}$ (see Fig. 8) or, what is equivalent, deviations from the Gaussian shape of the curves for large $R$ as well. Note, that for very small radii only a very small amount of particles will contribute to the pressure, i.e., at least, the central particle will contribute to the pressure.

These correlations vanish if we replace the forces in our data files by randomly and uniformly distributed forces. These (faked) data then show agreement with Eq. (7), see also Fig. 8, as expected, and finally confirm that correlations do occur in our systems (samples of particles that contribute to the pressure measured at the walls).

In a few systems, we also studied the correlations of the bulk-pressure in cubical volumes of different sizes (data not shown). The standard deviation of these data decays in close agreement with the expectations from the Central 
Limit Theorem. A more detailed study of the range of the correlations goes beyond the scope of this study, so that the open question why the correlations occur only at the walls remains open.

In order to check whether the correlations can be caused by the fact that our sampling areas (pressure-cells) have overlap, we studied the statistics with randomly leaving out 80 or 90 per-cent of the samples, with no significant change in the conclusions (besides some worse statistics).

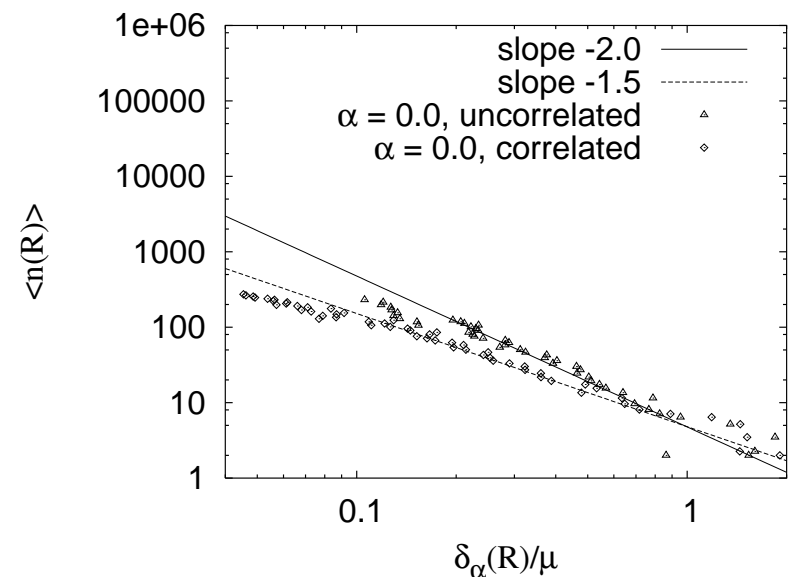

Figure 8: Number of particles averaged over many samples with given radius $R$, for many radii drawn from the interval of confidence for $\alpha=0$, scaled by the population mean. The simulations were performed with $N=20000$ particles and LSD. The data with slope of -1.5 obtained by our MD simulations reveal correlations between the forces of the wall-particles because they do not match to theory, see Eq. (7), that predicts a slope of -2 . Data with slope of -2 are "faked" by replacing the forces by uniformly distributed random forces.

\subsection{Correlation Function}

An alternative way to examine the correlations in a set of forces is to compute the correlation function

$$
C(r)=\frac{\left\langle f_{i} f_{j}(r)\right\rangle}{\left\langle f_{i}\right\rangle\left\langle f_{j}(r)\right\rangle},
$$

where the $f_{\alpha}$ are the forces of the center particles, for $\alpha=i$, and of all other particles at distance $r$ in the pressure cells, for $\alpha=j$. As displayed in Fig. 9 (Left), the correlations decay about two orders of magnitude within a distance 

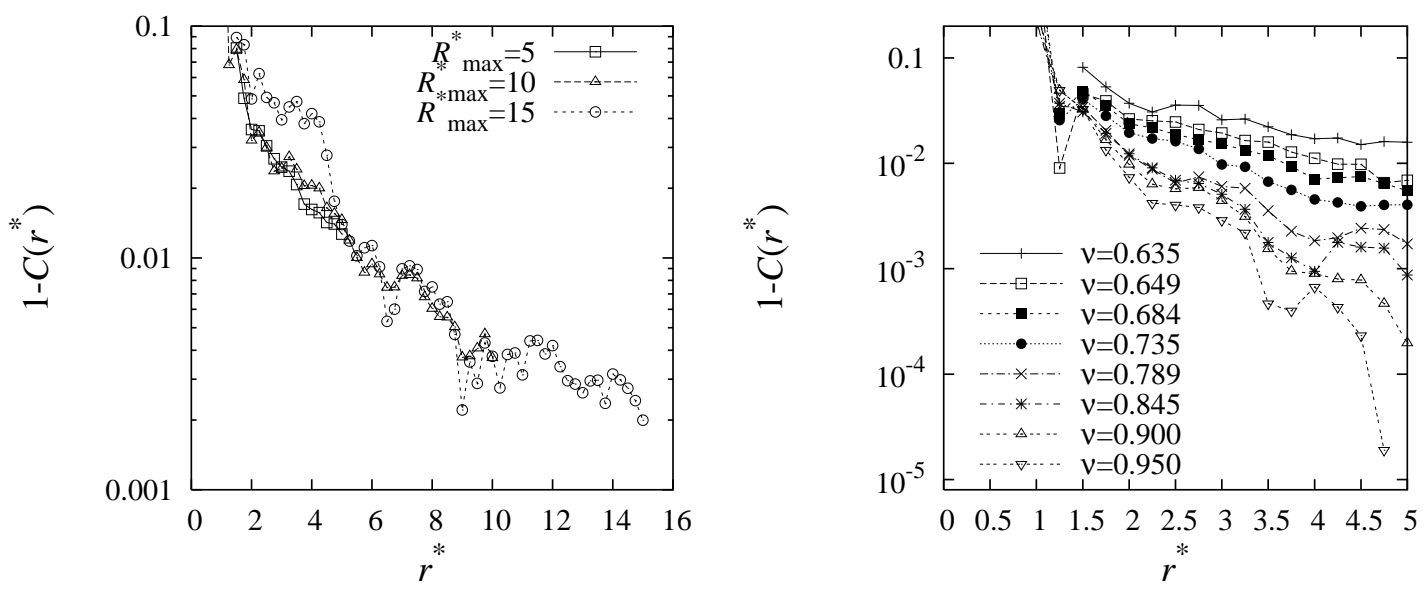

Figure 9: (Left) Correlation function $1-C\left(r^{*}\right)$ as function of the scaled distance from the center of the pressure cell, $r^{*}=r / r_{\max }$, for different sizes of the pressure cells $R_{\max }^{*}$, as given in the inset. (Right) Correlation function $1-C\left(r^{*}\right)$ for different densities $\nu$, as given in the inset.

of approximately 15 particle (maximal) radii. For small $R_{\max }^{*}$, this contrasts the results from the confidence interval (or standard deviation) measurements, where no change of behavior could be evidenced. These results do not change for larger $R_{\max }^{*}$.

The data for different densities in Fig. 9 (Right) show that the correlations decay much faster for larger densities. Note that there is no qualitative difference visible when one examines $\sigma / \mu_{P}$ as function of $R^{*}$ for different densities: The absolute values decrease with increasing density, but the slope of $-3 / 2$ remains independent of the density (data not shown). Thus, in conclusion, the pressure cell approach is capable of detecting a different type of correlations, which is not caught by the classical correlation function approach.

\section{Conclusions}

From static, frictionless and isotropically compressed packings, we extract the probability distribution of measured stresses on circular sensors at the walls. The width of the distribution decays more rapidly than expected from the Cen- 
tral Limit Theorem (CLT) for uncorrelated forces, which is in contrast to alternative previous observations $[31,32]$ on sheared, dynamic, and frictional particle systems.

Our main objective was to determine how the number of particles contributing to the pressure on a given sensor correlate with the interval of confidence, which is a measure for the width of the pressure density functions. As expected, the confidence interval increases the smaller the detector size is chosen, i.e., the worse the statistics becomes. Surprisingly, the relation $\langle n(R)\rangle \propto \delta_{\alpha}(R)^{-1.5}$ is observed, i.e. for a certain amount of particles, given a desired probability $1-\alpha$, the measured pressure values can be expected to be closer to the average, $\langle P(R)\rangle$, as assumed from CLT. Thus, our simulations predict a better confidence in measured data.

As a possible reason for this, one has the correlations between forces exerted by close-by particles on the walls. These correlations range over a rather long distance. The limited sensor size (and the related fluctuations of particle numbers) were excluded as source of this effect, because we found $\langle n(R)\rangle \propto \delta_{\alpha}(R)^{-2}$ for a fully random (uncorrelated), uniform force distribution.

Finally, we note that our systems were rather small, so that we cannot exclude the possibility that our observations are due to a finite size effect. Also the possibility of correlations due to the overlapping pressure cells cannot be excluded (avoiding the overlap leads to prohibitively bad statistics). Therefore, much larger simulations should be performed to confirm the present results and better understand the source of the correlations as evident from the width of the pressure probability density function.

Furthermore, we analysed our simulation data mainly at the walls, so that a direct experimental access to the same information is possible. Thus, the pressure correlations presented here should be confronted more systematically to bulk stress data (of both isotropic and deviatoric stresses) in order to learn if this is a wall effect or intrinsic also to bulk particulate systems, with force chains and the respective correlations, also far away from the walls.

Future studies should involve more realistic material properties like friction, 
and more realistic particle shapes as well as the study of cohesive, humid or saturated systems - all under dynamic driving or, e.g., shear.

\section{Acknowledgments}

Helpful discussions with K. Hopcraft and N. P. Kruyt are gratefully acknowledged. This work was made possible by the financial support of the Deutsche Forschungsgemeinschaft (DFG) and the Stichting voor Fundamenteel Onderzoek der Materie (FOM), financially supported by the Nederlandse Organisatie voor Wetenschappelijk Onderzoek (NWO). Furthermore, support from the Delft Platform for Compuational Science and Engineering (DCSE) is appreciated.

\section{References}

[1] C. Thornton, C. W. Randall, Applications of theoretical contact mechanics to solid particle system simulation, in: Micromechanics of granular media, Elsevier, Amsterdam, 1988.

[2] S. N. Coppersmith, C. Liu, S. Majumdar, O. Narayan, T. A. Witten, Model for force fluctuations in bead packs, Phys. Rev. E 53 (5) (1996) 4673-4685.

[3] F. Radjai, M. Jean, J. J. Moreau, S. Roux, Force distribution in dense two-dimensional granular systems, Phys. Rev. Lett. 77 (2) (1996) 274.

[4] F. Radjai, D. Wolf, S. Roux, M. Jean, J. J. Moreau, Force networks in granular packings, in: D. E. Wolf, P. Grassberger (Eds.), Friction, Arching and Contact Dynamics, World Scientific, Singapore, 1997.

[5] R. Brockbank, J. M. Huntley, R. Ball, Contact force distribution beneath a three-dimensional granular pile, J. Phys. II France 7 (1997) 1521-1532.

[6] M. E. Cates, J. P. Wittmer, J.-P. Bouchaud, P. Claudin, Jamming, force chains, and fragile matter, Phys. Rev. Lett. 81 (9) (1998) 1841-1844.

[7] F. Radjai, D. E. Wolf, The origin of static pressure in dense granular media, Granular Matter 1 (1998) 3-8. 
[8] F. Radjai, D. E. Wolf, M. Jean, J.-J. Moreau, Bimodal character of stress transmission in granular packings, Phys. Rev. Lett. 80 (1) (1998) 61-64.

[9] O. Tsoungui, D. Vallet, J.-C. Charmet, Use of contact area trace to study the force distributions inside 2d granular systems, Granular Matter 1 (2) (1998) 65-69.

[10] O. Tsoungui, D. Vallet, J.-C. Charmet, Dynamic effects on contact force distributions inside 2d granular materials, Phys. Rev. Lett.

[11] L. E. Silbert, D. E. s, G. S. Grest, T. C. Halsey, D. Levine, Geometry of frictionless and frictional sphere packings, Phys. Rev. E 65 (2001) 031304.

[12] D. L. Blair, N. W. Mueggenburg, A. H. Marshall, H. M. Jaeger, S. Nagel, Force distributions in 3d granular assemblies: Effects of packing order and inter-particle friction, Phys. Rev. E 63 (2001) 041304-1.

[13] J. H. Snoeijer, M. van Hecke, E. Somfai, W. van Saarloos, Force and weight distributions in granular media: Effects of contact geometry, Phys. Rev. E 67 (2003) 030302(R).

[14] P. T. Metzger, Granular contact force density of states and entropy in a modified edwards ensemble, Phys. Rev. E 70 (2004) 051303.

[15] J. H. Snoeijer, W. G. Ellenbroek, T. J. H. Vlugt, M. van Hecke, Sheared force-networks: anisotropies, yielding and geometry, cond-mat/0508411 (2005).

[16] C. Liu, S. R. Nagel, D. A. Schecter, S. N. Coppersmith, S. Majumdar, O. Narayan, T. A. Witten, Force fluctuations in bead packs, Science 269 (1995) 513.

[17] J. M. Erikson, N. W. Mueggenburg, H. M. Jaeger, S. R. Nagel, Force distribution in three-dimensional compressible granular packs, Phys. Rev. E 66 (2002) 040301(R). 
[18] B. P. Tighe, A. R. T. van Eerd, T. J. H. Vlugt, Entropy maximization in the network ensemble for granular solids, Phys. Rev. Lett. 100 (2008) 238001.

[19] A. Donev, I. Cisse, D. Sachs, E. Variano, F. H. Stillinger, R. Connelly, S. Torquato, P. M. Chaikin, Improving the density of jammed disordered packings using ellipsoids, Science 303 (5660) (2004) 990-993.

[20] E. I. Corwin, H. M. Jaeger, S. R. Nagel, Structural signature of jamming in granular media, Nature 435 (2005) 1075-1078.

[21] O. Dauchot, G. Marty, Dynamical heterogeneity close to the jamming transition in a sheared granular material, Phys. Rev. Lett. 95 (2005) 265701.

[22] C. Song, P. Wang, F. Potiguar, H. Makse, Experimental and computational studies of jamming, J. Phys.: Condens. Matter 17 (24) (2005) S2755-S2770.

[23] L. Silbert, A. J. Liu, S. R. Nagel, Vibrations and diverging lengthscales near the unjamming transition, Phys. Rev. Lett. 95 (2005) 098301.

[24] T. S. Majmudar, M. Sperl, S. Luding, R. P. Behringer, Jamming transition in granular systems, Phys. Rev. Lett. 98 (5) (2007) 058001.

[25] I. S. Aranson, L. S. Tsimring, F. Malloggi, E. Clément, Nonlocal rheological properties of granular flows near a jamming limit, Phys. Rev. E 78 (3) (2008) 031303.

[26] Z. Zeravcic, N. Xu, A. J. Liu, S. R. Nagel, W. van Saarloos, Excitations of ellipsoid packings near jamming, Europhys. Lett. 87 (2009) 26001.

[27] M. Mailman, C. F. Schreck, C. S. O’Hern, B. Chakraborty, Jamming in systems composed of frictionless ellipse-shaped particles, Phys. Rev. Lett. 102 (2009) 255501.

[28] C. E. Maloney, Correlations in the elastic response of dense random packings, Phys. Rev. Lett. 97 (2006) 035503. 
[29] A. Tanguy, J. Wittmer, F. Leonforte, J.-L. Barrat, Continuum limit of amorphous elastic bodies: A finite-size study of low-frequency harmonic vibrations, Physical Review B 66 (2002) 174205-1-174205-17.

[30] L. E. Silbert, M. Silbert, Long-wavelength structural anomalies in jammed systems, Phys. Rev. E 80 (2009) 041304.

[31] B. Miller, C. O’Hern, R. P. Behringer, Stress fluctuations for continuously sheared granular materials, Phys. Rev. Lett. 77 (1996) 3110.

[32] O. Baran, L. Kondic, Velocity profiles, stresses, and Bagnold scaling of sheared granular system in zero gravity, Phys. Fluids 17 (2005) 073304.

[33] O. Baran, L. Kondic, On velocity profiles and stresses in sheared and vibrated granular systems under variable gravity, Phys. Fluids 18 (2006) 121509.

[34] E. Jakeman, On the statistics of K-distributed noise, J. Phys. A: Math. Gen. 13 (1980) 31-48.

[35] D. M. Mueth, H. M. Jaeger, S. R. Nagel, Force distribution in a granular medium, Phys. Rev. E. 57 (3) (1998) 3164-3169.

[36] D. L. Blair, A. Kudrolli, Velocity correlations in dense granular gases, Phys. Rev. E 64 (5) (2001) 050301.

[37] M. P. Allen, D. J. Tildesley, Computer Simulation of Liquids, Oxford University Press, Oxford, 1987.

[38] S. Luding, Collisions \& contacts between two particles, in: H. J. Herrmann, J.-P. Hovi, S. Luding (Eds.), Physics of dry granular media - NATO ASI Series E350, Kluwer Academic Publishers, Dordrecht, 1998, p. 285.

[39] K. Bagi, Statistical analysis of contact force components in random granular assemblies, Granular Matter 5 (2003) 45-54. 